Revue Interventions économiques

Papers in Political Economy

$52 \mid 2015$

L'État social à l'épreuve de la crise financière de 2008

Pourquoi l'austérité? Perspectives comparées

Paul Vermeylen (2014). Le temps de la métropole. Agile, créative, solidaire, durable. Parcours en Europe. Paris : L'Harmattan. 286 pages

Diane-Gabrielle Tremblay

(2) OpenEdition

Édition électronique

URL : http://journals.openedition.org/interventionseconomiques/2511

DOI : 10.4000/interventionseconomiques.2511

ISBN : $1710-7377$

ISSN : $1710-7377$

Éditeur

Association d'Économie Politique

Référence électronique

Diane-Gabrielle Tremblay, «Paul Vermeylen (2014). Le temps de la métropole. Agile, créative, solidaire, durable. Parcours en Europe. Paris : L'Harmattan. 286 pages », Revue Interventions économiques [En ligne], 52 | 2015, mis en ligne le 01 mars 2015, consulté le 24 septembre 2020. URL : http:// journals.openedition.org/interventionseconomiques/2511; DOI : https://doi.org/10.4000/ interventionseconomiques.2511

Ce document a été généré automatiquement le 24 septembre 2020

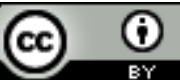

Les contenus de la revue Interventions économiques sont mis à disposition selon les termes de la Licence Creative Commons Attribution 4.0 International. 


\title{
Paul Vermeylen (2014). Le temps de la métropole. Agile, créative, solidaire, durable. Parcours en Europe. Paris : L'Harmattan. 286 pages
}

\author{
Diane-Gabrielle Tremblay
}

1 Ce livre nous permet de connaître tout un éventail d'expériences menées partout dans le monde, dans une diversité de villes. L'ouvrage part de l'idée selon laquelle, au sortir du fordisme, la nouvelle "économie de la connaissance ", telle qu'elle a été qualifiée, permettait aux territoires de se différencier par l'innovation technologique. On recherchait alors une plus grande valeur ajoutée par le biais de l'innovation technologique ou de produit. Puis, il y eut les délocalisations, la remise en question du mythe de la croissance continue, et le constat que nombre de pays en développement arrivaient à innover aussi bien que les pays industrialisés, d'où une remise en question des modalités du développement et de la croissance.

2 L'auteur soutient que les métropoles reprennent leur place comme source majeure d'innovation et de créativité. Les nouvelles métropoles « exemplaires » font davantage de place à la créativité que l'auteur qualifie de "fertilisante », affirmant que grâce au "frottement métropolitain", les aspirations des créateurs se trouvent en contact et s'alimentent les unes les autres, ce qui permet ultimement de satisfaire les attentes des habitants et des travailleurs. Pour plusieurs, les nouvelles attentes vont dans une direction différente de la croissance continue, allant plutôt vers des objectifs comme le développement durable, la créativité, l'agilité et la solidarité, autant de concepts en sous-titre à l'ouvrage.

3 L'ouvrage présente un ensemble de cas issus de diverses métropoles, et l'un des aspects fort intéressants du livre, c'est qu'on trouve à la fin une webographie qui nous permet d'approfondir la recherche sur ces cas et éventuellement de mettre à jour les 
observations. Autre aspect intéressant: nombre de cas mettent en évidence l'importance du dialogue et de la coopération pour une créativité efficace et un développement durable.

4 Le chapitre 1 traite de la métropole agile, et met notamment en avant des modes de gouvernance nouveaux, multiscalaires. La coopération entre régions et villes est ici mise de l'avant comme source de gouvernance nouvelle, l'ensemble des sous-sections fournissant une réflexion approfondie sur la gouvernance des métropoles.

5 Le chapitre 2 porte sur un thème très porteur depuis quelques années, à savoir la métropole créative. On met ici en évidence comment la créativité, l'intelligence, les talents peuvent donner lieu à une nouvelle forme de développement économique et à ce que l'auteur appelle une "prospérité rhizomique », qui se propage ou rayonne d'un secteur ou d'une ville à l'autre. Ici encore les vertus du dialogue et de la coopération sont mises en évidence, pour montrer comment les échanges et l'interaction peuvent favoriser une plus grande créativité. On rejoint ici les travaux des dernières années sur la ville créative, que ce soit ceux de Richard Florida, ou ceux de Charles Landry, mais il est intéressant de noter que le chapitre suivant traite d'une thématique souvent oubliée dans ces travaux, soit la question des inégalités et de la nécessaire solidarité entre les groupes.

6 Le chapitre 3 aborde ainsi le thème des liens sociaux, des meilleures pratiques à développer pour refonder le bien commun et remettre en marche ou renouveler les mouvements sociaux. Ici encore, l'annexe de fin donne un grand nombre de cas intéressants, qui vont de Newark aux États-Unis à Hambourg en Allemagne, en passant par Bruxelles, Dublin, Sofia ou encore Bucarest. Bref, un grand nombre de cas qui peuvent permettre de prolonger la lecture par une analyse de leur diversité à l'échelle internationale.

7 Le chapitre 4 porte sur la métropole durable et invite à adapter les processus de production et à réfléchir en termes d'écosystèmes. Les questions d'immobilier, de nature et de transition énergétique sont ici à l'ordre du jour, et l'engagement et la participation citoyenne sont aussi abordés dans cette perspective.

8 L'ouvrage se termine sur une conclusion qui invite vraiment à se centrer sur les métropoles, à réfléchir à la gouvernance politique de ces métropoles pour atteindre les objectifs évoqués dans les divers chapitres, dont le développement durable, la créativité, la solidarité et l'agilité.

9 L'ouvrage intéressera les chercheurs et étudiants en études urbaines, en sociologie, en géographie, ou en science politique, mais aussi les personnes qui interviennent activement en matière de politique locale ou municipale, voire à une échelle plus large.

L'ouvrage est très bien structuré, très bien écrit, de sorte que l'on peut y trouver un grand nombre d'idées pour alimenter l'action locale comme la recherche sur le rôle des villes, la créativité et l'ensemble des sujets traités dans ce livre. Alliant réflexion théorique et études de cas, l'ouvrage présente en tout cas des idées novatrices en matière de développement urbain. 


\section{AUTEUR}

\section{DIANE-GABRIELLE TREMBLAY}

Professeure, École des sciences de l'administration, Téluq-Université du Québec dgtrembl@teluq.ca 